\title{
Orange-to-red tunable picosecond pulses by frequency doubling in a diode-pumped PPKTP waveguide
}

\author{
K. A. Fedorova, ${ }^{1, *}$ G. S. Sokolovskii, ${ }^{1,2}$ D. I. Nikitichev, ${ }^{1}$ P. R. Battle, ${ }^{3}$ I. L. Krestnikov, ${ }^{4}$ \\ D. A. Livshits, ${ }^{4}$ and E. U. Rafailov ${ }^{1}$ \\ ${ }^{1}$ Photonics \& Nanoscience Group, School of Engineering, Physics and Mathematics, University of Dundee, Dundee DD1 4HN, UK \\ ${ }^{2}$ Ioffe Physico-Technical Institute, 26 Polytechnicheskaya str., St. Petersburg 194021, Russia \\ ${ }^{3}$ AdvR Inc., 2311 S. 7th Avenue, Building No. 1, Bozeman, Montana 59715, USA \\ ${ }^{4}$ Innolume GmbH, 11 Konrad-Adenauer-Allee, Dortmund 44263, Germany \\ ${ }^{*}$ Corresponding author: K.A.Fedorova@dundee.ac.uk
}

Received June 3, 2013; accepted July 1, 2013;

posted July 8, 2013 (Doc. ID 191690); published July 29, 2013

\begin{abstract}
A compact picosecond all-room-temperature orange-to-red tunable laser source in the spectral region between 600 and $627 \mathrm{~nm}$ is demonstrated. The tunable radiation is obtained by second-harmonic generation in a periodically poled potassium titanyl phosphate (PPKTP) multimode waveguide using a tunable quantum-dot external-cavity mode-locked laser. The maximum second-harmonic output peak power of $3.91 \mathrm{~mW}$ at $613 \mathrm{~nm}$ is achieved for $85.94 \mathrm{~mW}$ of launched pump peak power at $1226 \mathrm{~nm}$, resulting in conversion efficiency of $4.55 \%$. @ 2013 Optical Society of America

OCIS codes: (190.2620) Harmonic generation and mixing; (230.7370) Waveguides; (140.3538) Lasers, pulsed; (140.5960) Semiconductor lasers; (140.4050) Mode-locked lasers; (140.3600) Lasers, tunable.
\end{abstract}

http://dx.doi.org/10.1364/OL.38.002835

The development of compact and low-cost widely tunable coherent light sources emitting with picosecond pulse duration in the visible spectral region can provide indispensable tools for a variety of cutting-edge applications, such as biophotonics [1]], photomedicine [2- $\underline{4}]$, laser projection displays []ㅡ, confocal fluorescence microscopy $[\underline{6}, 7]$, etc. However, commercially available lasers of this spectral range are in practice bulky and inconvenient in use. An attractive approach for the realization of a compact visible laser source is the frequency doubling of an infrared laser diode in a nonlinear crystal containing a waveguide [8]. In this respect, the wide tunability [9] offered by quantum-dot external-cavity modelocked diode lasers (QD-ECMLLs), due to the broad gain bandwidth and the temperature insensibility $[10,11]$, is very promising for the development of a tunable visible source. Furthermore, QD materials have shown great promise for ultrafast technology, thus making QD lasers excellent candidates for versatile tunable ultrashort pulse pump sources [10] for second-harmonic generation (SHG) in the visible spectral region.

Over the last few years, great progress in material science, crystal growth, and semiconductor material processing in combination with recent advances in some of the more traditional technologies, in particular nonlinear frequency conversion, have led to the realization of a new generation of laser sources. Furthermore, the advent of a new generation of quasi-phase-matched waveguided nonlinear materials together with novel semiconductor lasers has led to the development of new frequency conversion sources with previously unattainable performance capabilities [12].

Recently, second- and third-harmonic generation in a periodically poled potassium titanyl phosphate (PPKTP) waveguide using a compact femtosecond Yb-based laser with conversion efficiencies of up to $33 \%$ (532 nm) and $2 \%(355 \mathrm{~nm})$, respectively, were demonstrated [13]. Green $(532 \mathrm{~nm})$ and yellow $(560 \mathrm{~nm})$ picosecond pulse sources that utilize SHG of Yb-doped fiber-based pump sources in PPKTP waveguides with maximum average powers of $41.7 \mu \mathrm{W}$ and $2.3 \mathrm{~mW}$, respectively, were also presented [14]. It has also been reported for a diodepumped femtosecond Cr:LiSAF laser (fundamental wavelength of $860 \mathrm{~nm}$ ) for which frequency doubling in a PPKTP waveguide afforded SH conversion efficiency of up to $37 \%$ [15]. Blue light generation at $488 \mathrm{~nm}$ in CW using a PPKTP waveguide crystal and high-brightness diode lasers with conversion efficiency of more than $260 \% / \mathrm{W}$ was also demonstrated [16]. Efficient blue light with maximum average power of $7.5 \mathrm{~mW}$ in picosecond pulses has been generated at 486, 488, and $491 \mathrm{~nm}$ from a frequency-doubled, nonresonant injection seeded, gainswitched InGaAs/GaAs diode laser by using a PPKTP waveguide with a Bragg grating section [17]. Recently, a frequency-doubling scheme generating $4.3 \mathrm{~mW}$ of orange light at $613 \mathrm{~nm}$ with conversion efficiency exceeding $10 \%$ from a PPKTP waveguide end pumped by a CW QD diode laser was also reported [8].

Here we present a compact all-room-temperature broadly tunable visible laser source in the picosecond regime (between 600 and $627 \mathrm{~nm}$ ) by SHG in a PPKTP waveguide using a widely tunable QD-ECMLL. The approach used in our work for demonstration of the tunable SHG is based on the utilization of a significant difference in the effective refractive indices of the high-order and low-order modes in the waveguide [12], which enables one to shift the difference between the effective refractive indices of the fundamental and second-harmonic waves to match the period of poling in the very broad wavelength range limited mainly by the waveguide refractive index step.

The experimental setup was similar to that described in [12] and consisted of a QD gain chip and a PPKTP waveguide, as shown in Fig. 1. The QD gain chip had a total length of $4 \mathrm{~mm}$ with a $1 \mathrm{~mm}$ absorber. The ridge waveguide was angled at $7^{\circ}$ relative to the normal of the 


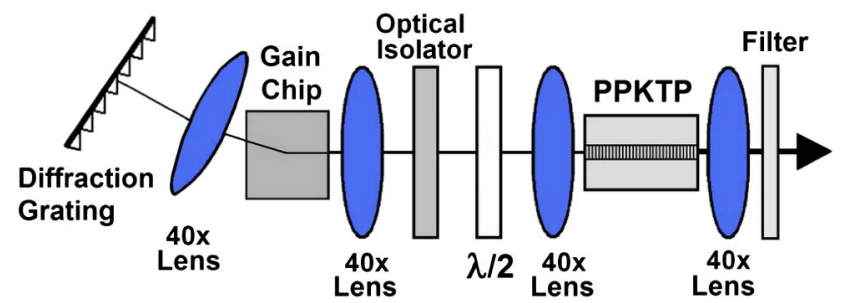

Fig. 1. Schematic of the experimental setup.

antireflective (AR) coated back facet (both facets had conventional AR coatings, resulting in total estimated reflectivities of $10^{-2}$ for the front facet and less than $10^{-5}$ for the angled facet). The QD gain chip was set up in a quasi-Littrow configuration, whereby the radiation emitted from the back facet of the chip was coupled onto the diffraction grating (1200 grooves/mm), which reflected the first-order of the diffracted beam back to the gain chip [11]. Coarse wavelength tuning of the QD-ECMLL between 1193 and $1284 \mathrm{~nm}$ in the picosecond regime at $20^{\circ} \mathrm{C}$ was possible for pump current of $900 \mathrm{~mA}$ and reverse bias of $3 \mathrm{~V}$. Pulsed operation was observed at any wavelength. The laser output after an optical isolator was coupled using an aspheric lens $(\mathrm{NA} \sim 0.55)$ into the PPKTP waveguide.

The waveguide was fabricated by the ion-exchange technique [18], which provided a refractive index step of $\Delta \mathrm{n} \approx 0.01$. With this technique, the masked KTP crystal was immersed in an ion-exchange bath consisting of molten nitrate salts of $\mathrm{Rb}\left(\mathrm{RbNO}_{3}\right)$ [19]. Within this bath, the $\mathrm{Rb}$ ions diffused through a mask into the substrate, while the K ions diffused out of the KTP crystal. In the diffused regions, the $\mathrm{Rb}$ ions increased the refractive index relatively to the undiffused KTP and thus formed the optical waveguide. The periodic poling was performed after the waveguides were fabricated using an applied electric field to periodically invert the domains.

The PPKTP frequency-doubling crystal waveguide (not AR coated) used in our work was $13 \mathrm{~mm}$ in length with a cross-sectional area of $\sim 4 \mu \mathrm{m} \times 4 \mu \mathrm{m}$ and was periodically poled for SHG at $\sim 1226 \mathrm{~nm}$ (with the poling period of $\sim 11.9 \mu \mathrm{m}$ ). Both the pump laser and the PPKTP crystal were operating at room temperature. The frequency-doubled output light was then collected by a power meter after a suitable filter at the fundamental wavelength.

The calculated dependence of the poling period on wavelength (Fig. 2) illustrates the SHG tunability in a multimode PPKTP waveguide with a refractive index step of $\Delta n=0.01$. Details of the model that takes into account the difference of the effective refractive indices of the high- and low-order modes are described elsewhere [12]. The horizontal gray line represents the physical poling period $(11.9 \mu \mathrm{m})$ of the crystal used in the experiment, the orange bold line is the central dispersion curve (crossing the gray line at the designed wavelength of $1226 \mathrm{~nm}$ ), and the blue and red lines are the SHG tunability margins for the waveguide refractive index step of $\sim 0.01$.

Tunable SHG in the spectral region between 600 and $627 \mathrm{~nm}$ was demonstrated (Fig. 3) in the pulsed operation with repetition rate between 2.64 and $7.92 \mathrm{GHz}$ and pulse

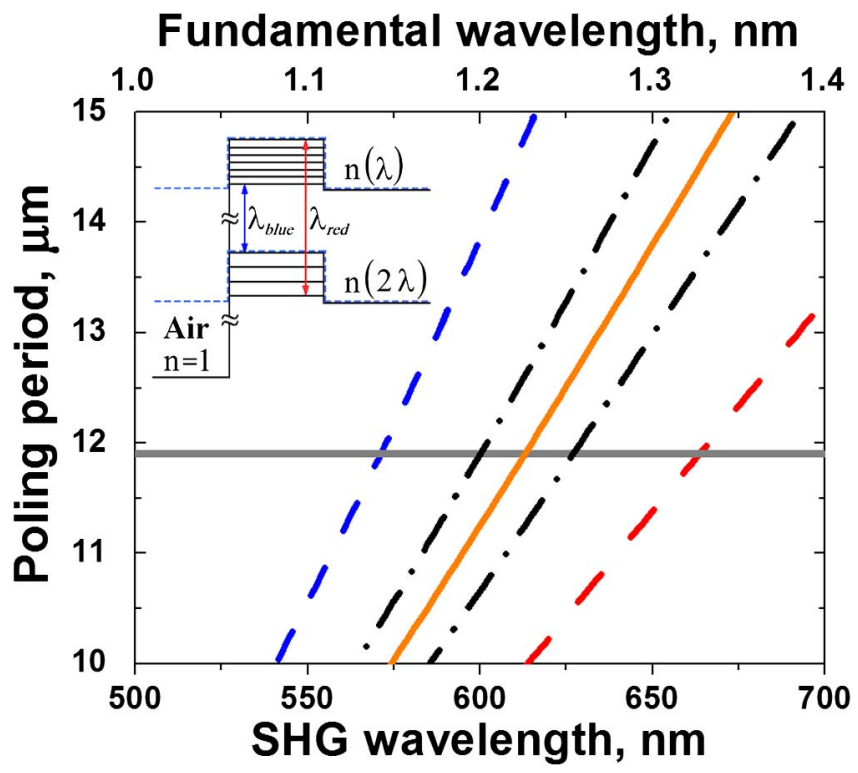

Fig. 2. Dependence of the poling period on wavelength for different-order waveguide modes for frequency doubling in a PPKTP waveguide. Inset: simplified schematic of the effective refractive indices for the fundamental and $\mathrm{SH}$ modes of different order. The refractive index profiles of the waveguide in two orthogonal planes are shown by the solid and dashed lines.

duration between 14.7 and 29.3 ps. The maximum SHG output peak power of $3.91 \mathrm{~mW}$ at $613 \mathrm{~nm}$ was achieved at $6.16 \mathrm{GHz}$ repetition rate and $15.3 \mathrm{ps}$ pulse duration (Fig. 4). The corresponding maximum SHG output and launched average powers were $369 \mu \mathrm{W}$ and $8.1 \mathrm{~mW}$, respectively, resulting in conversion efficiency of $4.55 \%$ in the picosecond regime. Meanwhile, SHG in the same crystal waveguide in the $\mathrm{CW}$ regime demonstrated only $2.14 \%$ of the conversion efficiency, which corresponded to $173 \mu \mathrm{W}$ of the SHG output average power at $613 \mathrm{~nm}$ obtained from the same launched average pump power [12]. This can be explained by the higher peak power used for the picosecond SHG presented here.

Frequency doubling at 600 and $627 \mathrm{~nm}$, which corresponded to phase matching between fundamental and

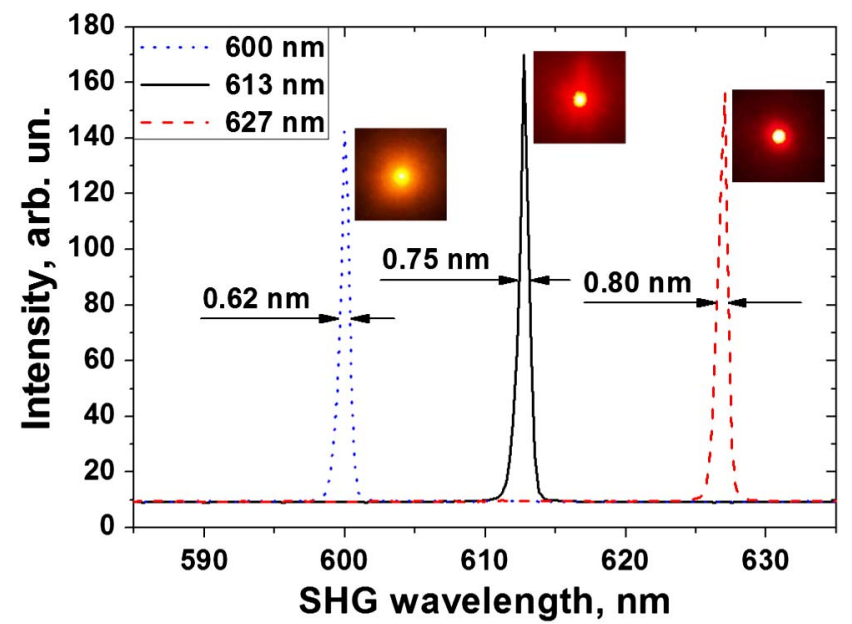

Fig. 3. Optical spectra of the SHG light at 600, 613, and $627 \mathrm{~nm}$. Spectral widths are limited by the instrumental resolution of the WaveScan spectrometer. 


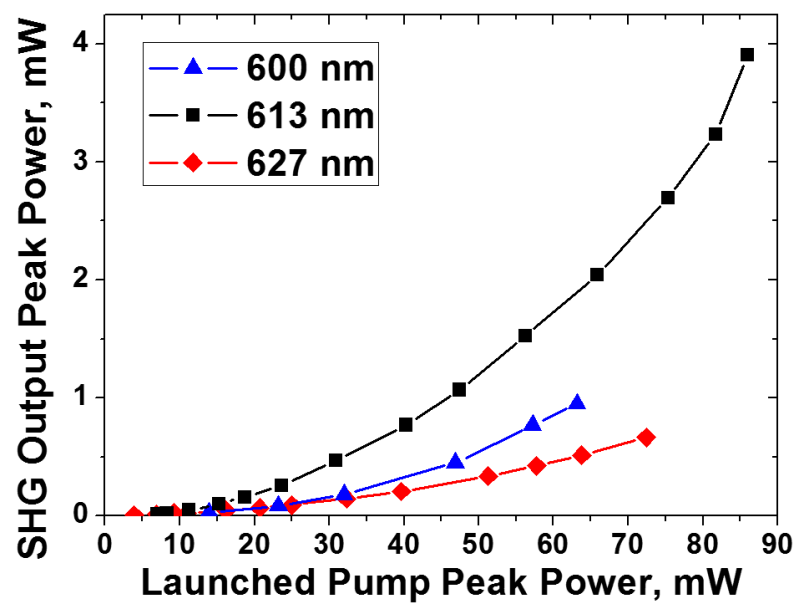

Fig. 4. Frequency-doubled output peak power versus launched pump power at 600,613, and $627 \mathrm{~nm}$.

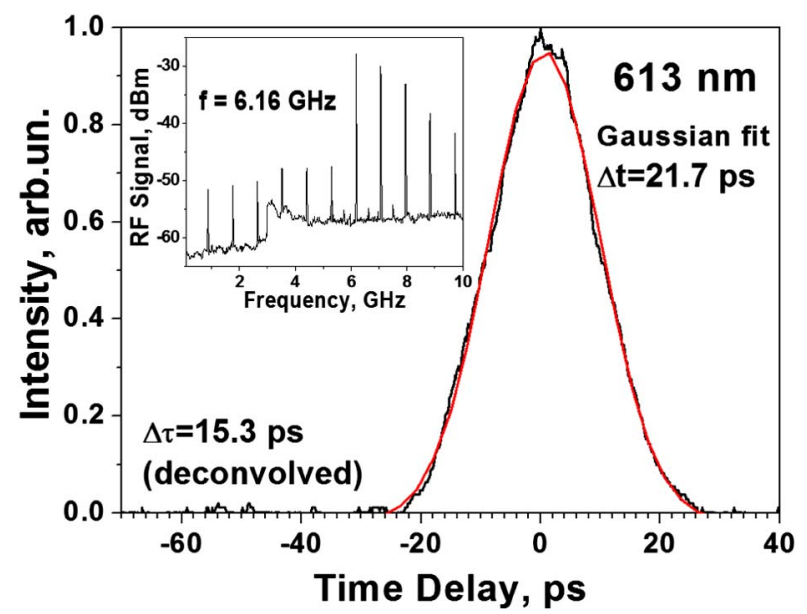

Fig. 5. Autocorrelation signal at $613 \mathrm{~nm}$ for $900 \mathrm{~mA}$ of gain current and $3 \mathrm{~V}$ of reverse bias. Insert: corresponding $\mathrm{RF}$ spectrum.

SHG modes of different order, was demonstrated with output peak power of 0.95 and $0.66 \mathrm{~mW}$ and with conversion efficiency of $1.5 \%$ and $0.92 \%$, respectively. RF spectrum and autocorrelation signal corresponding to the SHG at $613 \mathrm{~nm}$ are shown in Fig. 5 .

To summarize, we have demonstrated a compact tunable all-room-temperature picosecond laser source in the visible spectral region (between 600 and $627 \mathrm{~nm}$ ) with the maximum second-harmonic output peak power of $3.91 \mathrm{~mW}$ and conversion efficiency of $4.55 \%$ at $613 \mathrm{~nm}$. This system is based on SHG using a single QD modelocked diode laser and a single PPKTP waveguide and represents a practical laser source that is of high interest for a wide range of applications. The demonstrated results, which are based on the different-order mode interaction in the SHG waveguide, open the preferred way for realization of a compact full-color laser source. The work on improvement of SHG conversion efficiency and further extension of operation to different spectral regions is currently underway.

This work was partly supported by the EU FP7 program through a FAST-DOT project (contract no. 224338).

\section{References}

1. W. G. Telford, Methods Cell. Biol. 102, 375 (2011).

2. S. Choudhary, K. Nouri, and L. Elsaie, Lasers Med. Sci. 24, 971 (2009).

3. L. Baratto, L. Calza, R. Capra, M. Gallamini, L. Giardino, A. Giuliani, L. Lorenzini, and S. Traverso, Lasers Med. Sci. 26, 103 (2011).

4. A. Klein, W. Baumler, M. Landthaler, and P. Babilas, Lasers Med. Sci. 26, 845 (2011).

5. M. Jansen, G. P. Carey, R. Carico, R. Dato, A. M. Earman, M. J. Finander, G. Giaretta, S. Hallstein, H. Hofler, C. P. Kocot, S. Lim, J. Krueger, A. Mooradian, G. Niven, Y. Okuno, F. G. Patterson, A. Tandon, and A. Umbrasas, Proc. SPIE 6489, 648908 (2007).

6. M. Mueller, Introduction to Confocal Fluorescence Microscopy, Tutorial Texts in Optical Engineering, Vol. TT69 (SPIE, 2006).

7. N. S. Claxton, T. J. Fellers, and M. W. Davidson, "Laser scanning confocal microscopy," http://olympusfluoview .com/theory/LSCMIntro.pdf.

8. K. A. Fedorova, M. A. Cataluna, P. R. Battle, C. M. Kaleva, I. L. Krestnikov, D. A. Livshits, and E. U. Rafailov, Appl. Phys. B 103, 41 (2011).

9. D. I. Nikitichev, K. A. Fedorova, Y. Ding, A. Alhazime, A. Able, W. Kaenders, I. Krestnikov, D. Livshits, and E. U. Rafailov, Appl. Phys. Lett. 101, 121107 (2012).

10. E. U. Rafailov, M. A. Cataluna, and W. Sibbett, Nat. Photonics 1, 395 (2007).

11. K. A. Fedorova, M. A. Cataluna, I. Krestnikov, D. Livshits, and E. U. Rafailov, Opt. Express 18, 19438 (2010).

12. K. A. Fedorova, G. S. Sokolovskii, P. R. Battle, D. A. Livshits, and E. U. Rafailov, Laser Phys. Lett. 9, 790 (2012).

13. M. Rusu, E. U. Rafailov, R. Herda, O. G. Okhotnikov, S. M. Saltiel, P. Battle, S. McNeil, A. B. Grudinin, and W. Sibbett, Appl. Phys. Lett. 88, 121105 (2006).

14. D. A. Chestnut, S. V. Popov, J. R. Taylor, and T. D. Roberts, Appl. Phys. Lett. 88, 071113 (2006).

15. B. Agate, E. U. Rafailov, W. Sibbett, S. M. Saltiel, K. Koinov, M. Tihonen, S. Wang, F. Laurell, P. Battle, T. Fry, T. Roberts, and E. Noonan, IEEE J. Sel. Top. Quantum Electron. 10, 1268 (2004).

16. A. Jechow, S. McNeil, C. Kaleva, D. Skoczowsky, and R. Menzel, Proc. SPIE 7197, 719707 (2009).

17. E. U. Rafailov, D. J. L. Birkin, W. Sibbett, P. Battle, T. Fry, and D. Mohatt, Opt. Lett. 26, 1961 (2001).

18. J. D. Bierlein, A. Ferretti, L. H. Brixner, and W. Y. Hsu, Appl. Phys. Lett. 50, 1216 (1987).

19. B. Agate, E. U. Rafailov, W. Sibbett, S. M. Saltiel, K. Koynov, M. Tiihonen, S. Wang, F. Laurell, P. Battle, T. Fry, T. Roberts, and E. Noonan, in Frontiers in Planar Lightwave Circuit Technology, Vol. 216 of NATO Science Series II: Mathematics, Physics And Chemistry (Springer, 2006), pp. 189-227. 